\title{
ADAPTIVE FILTERS
}

\section{Ali H. Sayed}

Electrical Engineering Department

University of California, Los Angeles

A. H. Sayed, Adaptive Filters, Wiley, NJ, 2008.

Readers are welcome to bring to the attention of the author any typos or suggestions for improvements. Please feel free to email the author at sayed@ee.ucla.edu.

The author is thankful for your feedback.

The author is particularly thankful to Mr. M. Tsakiris for a list of typos. 


\section{ERRATA}

Last updated November 2015

\section{CHAPTERS}

\section{Appendix B}

- Page 15, equation (B.1): remove "and $B>0$ " from the left-hand side.

\section{Appendix C}

- Page 27, first sentence under Hessian Matrix, replace "The complex gradient of $\nabla_{z} g$ with respect to $z^{*}$ is called..." by "When $g(z)$ is quadratic in $z$, the complex gradient of $\nabla_{z} g$ with respect to $z^{*}$ determines..."

\section{Chapter 2}

- Page 45, Example 2.2, first line: replace "Ex. A.4” by "Ex. A.2."

\section{Chapter 4}

- Page 69, Example 4.3, first line: replace "Ex. A.4" by "Ex. A.2."

- Page 70, Example 4.4, first line: replace "Ex. 4.1" by "Ex. 4.3."

\section{Chapter 7}

- Page 108, prior to last equation on page: replace $\left(H_{i} \tilde{\boldsymbol{x}}_{i \mid i-1}+0\right.$ by $\left(H_{i} \tilde{\boldsymbol{x}}_{i \mid i-1}\right)^{*}+0$.

\section{Chapter 9}

- Page 158, last sentence of Theorem 9.1: replace "divergent sequence" by "divergent series."

\section{Chapter 13}

- Page 194, after (13.10): replace "the equality $d_{i}=u_{i} w_{i}$ " by "the equality $d_{i}=U_{i} w_{i}$ "

- Page 196, statement of Alg. 13.2: replace $\left\|\tilde{u}_{i-j+k}\right\|^{2}$ by $\left\|\tilde{u}_{i-k+j}\right\|^{2}$ and $\tilde{u}_{i-k}$ by $\tilde{u}_{i-k}^{*}$.

\section{Chapter 15}

- Page 241, equation (15.45): in the expression for $\sin ^{2}\left(\theta_{i}\right)$, replace $\left\|\tilde{\boldsymbol{w}}_{i-1}\right\|^{2}$ by $\left\|\tilde{\boldsymbol{w}}_{i}\right\|^{2}$ here and in the earlier expression on the same page.

\section{Chapter 19}

- Page 269, Table 19.1, the expression for the EMSE of $\epsilon$-NLMS with power normalization in the third row of the table should be replaced by (adjust the denominator):

$$
\zeta^{\epsilon-\mathrm{pNLMS}}=\frac{\mu(1+\beta) M \sigma_{v}^{2}}{2[\gamma(1-\beta)+2 \beta]-\mu M(1+\beta)}
$$

\section{Chapter 21}

- Page 286, equation (21.29): replace $\mu \operatorname{Tr}\left(R_{u}\right)$ by $2 \mu \operatorname{Tr}\left(R_{u}\right)$.

- Page 295, Table 21.2, the expression for the EMSE of $\epsilon$-NLMS with power normalization in the third row of the table should be replaced by (adjust the denominator):

$$
\zeta^{\epsilon-\mathrm{pNLMS}}=\frac{\mu(1+\beta) M \sigma_{v}^{2}+\mu^{-1} \gamma \sigma_{u}^{2}(1-\beta) \operatorname{Tr}(Q)}{2[\gamma(1-\beta)+2 \beta]-\mu M(1+\beta)}
$$

\section{Chapter 27}

- Page 428, equation (27.5), adjust the indices of the entries in $u_{B, n}$ and $y_{B, n}$ to:

$$
u_{B, n} \triangleq\left[\begin{array}{c}
u(n B) \\
u(n B-1) \\
\vdots \\
u((n-1) B+1)
\end{array}\right], \quad y_{B, n} \triangleq\left[\begin{array}{c}
y(n B) \\
y(n B-1) \\
\vdots \\
y((n-1) B+1)
\end{array}\right]
$$


The same adjustment should be made in the statement of Algorithm 27.1 on page 438 and in other locations in the chapter listed next.

- Page 429, adjust the indices in the top two expressions to:

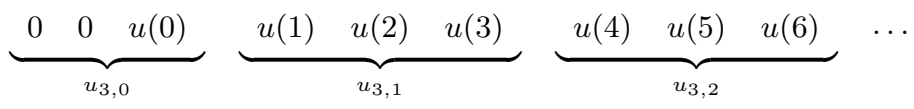

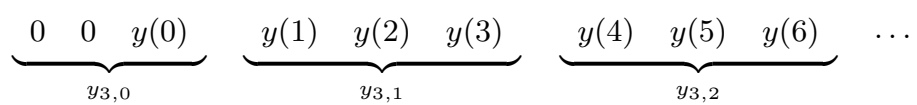

- Page 433, adjust the entries in the vector after equation (27.22) to

$$
\left[\begin{array}{l}
u(6) \\
u(5) \\
u(4) \\
\hline u(3) \\
u(2) \\
u(1)
\end{array}\right]
$$

- Page 435 , replace the indices in the first expression by

$$
y_{B, 2}=\left[\begin{array}{l}
y(6) \\
y(5) \\
y(4)
\end{array}\right], \quad y_{B, 1}=\left[\begin{array}{l}
y(3) \\
y(2) \\
y(1)
\end{array}\right], \quad y_{B, 0}=\left[\begin{array}{c}
y(0) \\
0 \\
0
\end{array}\right]
$$

and adjust the entries in the three rows below it accordingly.

- Page 435, Figure 27.7, the entries on the left of the figure from top to bottom should likewise be $\{y(n B), \ldots, y((n-1) B+2), y((n-1) B+1)\}$.

\section{Chapter 28}

- Page 440, first equation, adjust the indices of the entries in $d_{B, n}$ and $v_{B, n}$ to:

$$
d_{B, n} \triangleq\left[\begin{array}{c}
d(n B) \\
d(n B-1) \\
\vdots \\
d((n-1) B+1)
\end{array}\right], \quad v_{B, n} \triangleq\left[\begin{array}{c}
v(n B) \\
v(n B-1) \\
\vdots \\
v((n-1) B+1)
\end{array}\right]
$$

Adjust the indices accordingly in the statements of Algorithms 28.1, 28.2, 28.3, 28.4, 28.5, 28.6, and 28.7 on pages $442,445,455,459,460,461$, and 462 for the above two vectors and similarly for $u_{B, n}, \hat{d}_{B, n}, e_{B, n}$, and $y_{B, n}$ :

$$
\begin{aligned}
& u_{B, n}=\operatorname{col}\{u(n B), u(n B-1), \ldots, u((n-1) B+1)\} \\
& \hat{d}_{B, n}=\operatorname{col}\{\hat{d}(n B), \hat{d}(n B-1), \ldots, \hat{d}((n-1) B+1)\} \\
& e_{B, n}=\operatorname{col}\{e(n B), e(n B-1), \ldots, e((n-1) B+1)\} \\
& y_{B, n}=\operatorname{col}\{y(n B), y(n B-1), \ldots, y((n-1) B+1)\}
\end{aligned}
$$

- Page 454 , replace $n B+j$ by $n B-j$ in the indices in equation (28.20) and the line after it. Likewise, replace $\left\{\hat{d}(n B+j), u_{n B+j}\right\}$ on that page by $\left\{\hat{d}(n B-j), u_{n B-j}\right\}$. The same adjustments should be made in the statements of Algorithms 28.3, 28.7 on pages 455, 462.

\section{Chapter 29}

- Page 482, second line below (29.16): replace "we multiply (29.16) from the left" by "we multiply (29.16) by $H^{*}$ from the left".

\section{Chapter 32}


- Page 516, second line after second equation from top: replace $\mathcal{R}(y)$ by $\mathcal{R}(H)$.

- Page 524, first equation on top: the locations of the upper and lower triangular matrices involving $\widehat{w}^{b}$ should be exchanged.

- Page 525, second equality for the expression for $\gamma_{z}$ : same as above.

- Page 529, right above (32.48): replace $(N-1) \times M$ by $N \times M$.

\section{Chapter 33}

- Page 565, first sentence in Lemma 33.1: replace "Given" by "Consider".

- Page 570: replace "App. 34” by "Chapter 34."

\section{Chapter 34}

- Page 578, second paragraph under Complex data: replace $z_{o}$ by $z(0)$.

\section{Chapter 35}

- Page 585: replace "App. 34" by "Chapter 34."

\section{Chapter 37}

- Page 615: in the second and third equations after (37.18), the quantities $C$ and $D$ should be replaced by $D$ and $E$, respectively.

\section{Chapter 40}

- Page 659, equation (40.34), replace $\lambda^{i} w^{\bar{b} *} \Pi_{2} w^{\bar{b}}$ by $\lambda^{i} w_{2}^{\bar{b} *} \Pi_{2} w_{2}^{\bar{b}}$.

\section{Chapter 41}

- Page 674, Figure 41.3: the time index for the reflection coefficients in the figure should be $(i-1)$ instead of $(i)$.

\section{Chapter 43}

- Page 694, Algorithm 43.1, step 1: add the initial condition $\zeta_{m}^{b / 2}(-2)=\sqrt{\eta^{-1} \lambda^{-m-3}}$.

\section{Chapter 44}

- Pages 707-709, Figures 44.1-44.3: due to a production error, these figures appear distorted. For your reference, the actual figures are reproduced in subsequent pages in this file.

\section{PROBLEMS}

\section{Part II}

- Page 116, Problems II.8, II.9, II.10: replace "Ex. 4.1” by "Ex. 4.3."

\section{Part III}

- Page 210, Problem III.9, part (a): replace $y \geq 0$ by $0 \leq y<1$.

- Page 211, Problem III.11, part (a): replace $2+\epsilon / \lambda_{\max }$ by $2+2 \epsilon / \lambda_{\max }$.

- Page 212, Problem III.16, part (b): replace $\sigma_{d}=\mathrm{E} \boldsymbol{d}^{2}$ by $\sigma_{d}^{2}=\mathrm{E} \boldsymbol{d}^{2}$.

- Page 213, Problem III.18: replace "constant number $\gamma$ " by "positive number $\gamma$ ".

- Page 220, Problem III.44, second paragraph: replace $\left\{u_{i}, u_{i-2}, u_{i-3}\right\}$ by $\left\{u_{i}, u_{i-1}, u_{i-2}\right\}$. In part (a), remove the ... from inside diag.

\section{Part IV}

- Page 306, Problem IV.5, parts (b) and (c):

$$
\begin{aligned}
\mathrm{E} \boldsymbol{p}^{2}(i) & =\gamma \sigma_{u}^{4}\left(1-\beta^{2(i+1)}\right) \frac{1-\beta}{1+\beta}+2 \sigma_{u}^{4}\left(1-\beta^{i+1}\right)\left(1-\beta^{i}\right) \frac{\beta}{1+\beta} \\
\zeta^{\epsilon-\mathrm{pNLMS}} & =\frac{\mu(1+\beta) M \sigma_{v}^{2}}{2[\gamma(1-\beta)+2 \beta]-\mu M(1+\beta)}
\end{aligned}
$$

- Page 309, Problem IV.11, replace reference to "Prob. III.15" by "Prob. IV.10." 
- Page 314, Problem IV.23, part (a): replace $4 \eta_{u}^{2} \sigma_{v}^{2}$ by $4 \eta_{u}^{2} \sigma_{v}^{4}$.

- Page 314, Problem IV.24, top of page 315, update the expression in the denominator:

$$
\zeta^{\epsilon-\mathrm{pNLMS}}=\frac{\mu(1+\beta) M \sigma_{v}^{2}+\mu^{-1} \gamma \sigma_{u}^{4}(1-\beta) \operatorname{Tr}(Q)}{2[\gamma(1-\beta)+2 \beta]-\mu M(1+\beta)}
$$

- Page 325, part (e): replace "Use the last 5600 of the signals.." by "Use the last 5600 samples of the signals.."

\section{Part VI}

- Page 470, Problem VI.12, replace $+i$ by $-i$ in the subscripts and indices in the four equations on top of the page.

- Page 470, Problem VI.13, replace $\left\{\boldsymbol{u}_{n B+i}, \boldsymbol{d}(n B+i)\right\}$ by $\left\{\boldsymbol{u}_{n B-i}, \boldsymbol{d}(n B-i)\right\}$ and the indices in $\left\{\boldsymbol{U}_{n}, \boldsymbol{e}_{B, n}\right\}$ by:

$$
\boldsymbol{U}_{n}=\left[\begin{array}{c}
\boldsymbol{u}_{n B} \\
\boldsymbol{u}_{n B-1} \\
\vdots \\
\boldsymbol{u}_{(n-1) B+1}
\end{array}\right], \quad \boldsymbol{e}_{B, n}=\left[\begin{array}{c}
\boldsymbol{e}(n B) \\
e(n B-1) \\
\vdots \\
\boldsymbol{e}((n-1) B+1)
\end{array}\right]
$$

\section{Part VII}

- Page 541, Problem VII.7, replace $\left\|d_{i}-U_{i} w_{i-1}\right\|^{2}$ by $\left\|d_{i}-U_{i} w_{i}\right\|^{2}$.

\section{Part X}

- Page 699, Problem X.7, step 1: add under initial conditions $c_{m}^{b}(-1)=1, s_{m}^{b}(-1)=0$, $b_{m}^{\prime}(-1)=0$. 


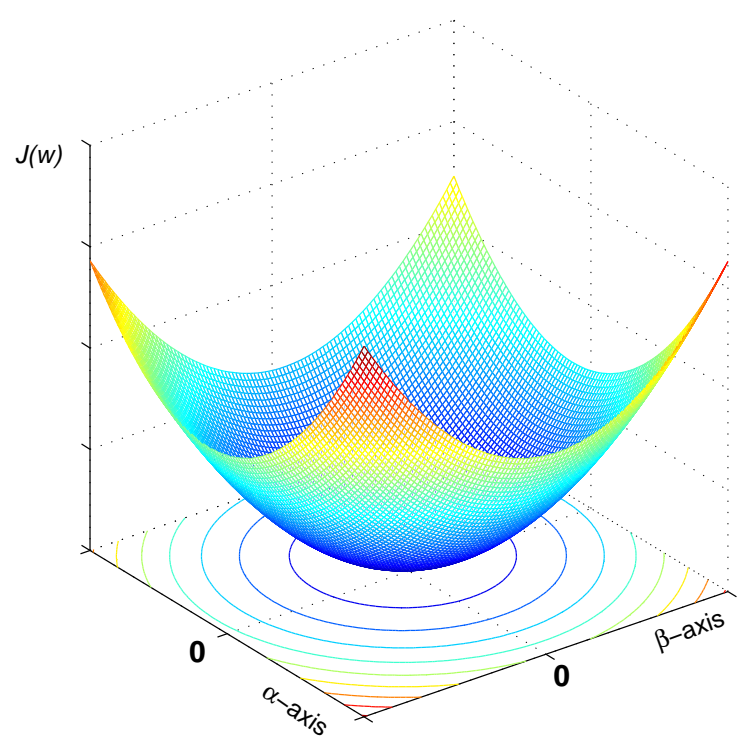

FIGURE 44.1 A typical plot of a quadratic cost function $J(w)$ with a global minimum for the case in which $w$ is two-dimensional, say, $w=\operatorname{col}\{\alpha, \beta\}$. The plot also shows the contour curves of $J(w)$. 


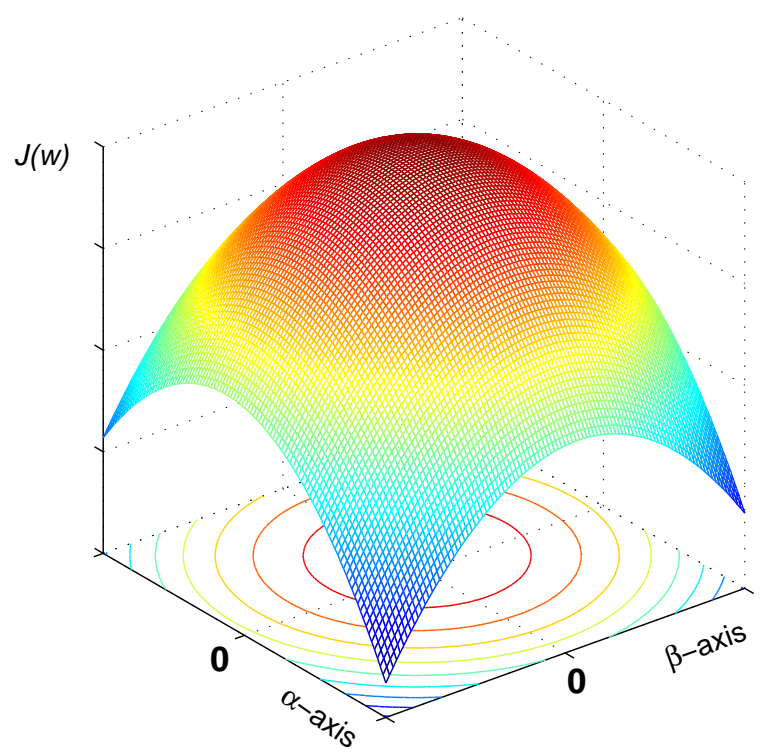

FIGURE 44.2 A typical plot of a quadratic cost function $J(w)$ with a global maximum for the case in which $w$ is two-dimensional, say, $w=\operatorname{col}\{\alpha, \beta\}$. The plot also shows the contour curves of $J(w)$. 


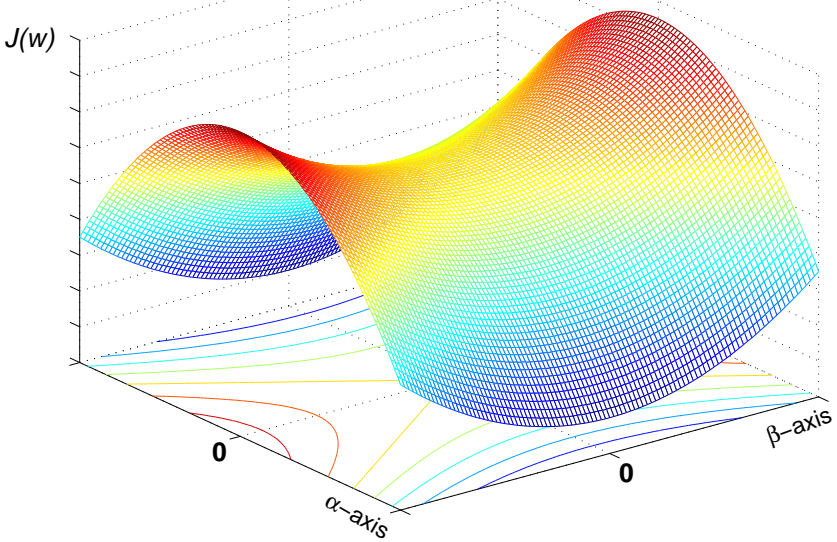

FIGURE 44.3 A typical plot of a quadratic cost function $J(w)$ with a saddle point for the case in which $w$ is two-dimensional, say, $w=\operatorname{col}\{\alpha, \beta\}$. The plot also shows the contour curves of $J(w)$. 